Revue d'histoire de l'Amérique française

ZAS REVUE D.HISTOIRE DE L'AMÉRIQUE FRANÇAISE

\title{
Les évêques du Bas-Canada et le projet d'Union (1840)
}

\section{Léon Pouliot}

Volume 8, numéro 2, septembre 1954

URI : https://id.erudit.org/iderudit/301647ar

DOI : https://doi.org/10.7202/301647ar

Aller au sommaire du numéro

Éditeur(s)

Institut d'histoire de l'Amérique française

ISSN

0035-2357 (imprimé)

1492-1383 (numérique)

Découvrir la revue

Citer cet article

Pouliot, L. (1954). Les évêques du Bas-Canada et le projet d'Union (1840). Revue d'histoire de l'Amérique française, 8(2), 157-170.

https://doi.org/10.7202/301647ar d'utilisation que vous pouvez consulter en ligne.

https://apropos.erudit.org/fr/usagers/politique-dutilisation/ 


\section{LES EVEQQUES DU BAS-CANADA ET LE PROJET D'UNION (1840)}

L'Union des Canadas étant, de sa nature, un projet politique, on ne doit pas s'attendre à voir les évêques prendre la tête d'un mouvement d'opposition ou d'approbation. Comme citoyens, ils ont droit à leur opinion et ils ont même le droit de la manifester. Mais la loi du plus grand bien leur interdit de le faire avec éclat. Le prêtre et l'évêque sont hommes d'Église avant d'être citoyens de l'Etat; et dans le cours ordinaire des choses, leur participation active à la politique amènerait à confondre le citoyen et l'homme d'Eglise, au détriment de celui-ci.

Mais un projet politique comme celui de l'Union des Canadas n'est pas sans affecter d'une manière ou d'une autre les droits, libertés et privilèges de l'Église, dont les évêques sont les gardiens. Et ici, ils ont non seulement le droit, ils ont le devoir d'intervenir. Leur mode d'intervention doit rester digne des intérêts supérieurs qu'ils ont à défendre et du caractère sacré dont ils sont revêtus. Après l'exposé de ces principes, venons-en aux faits.

En 1840, il y a quatre évêques dans le Bas-Canada: Mgr Signay et son coadjuteur, Mgr Turgeon, à Québec; Mgr Lartigue et son coadjuteur, Mgr Bourget, à Montréal. Nous savons avec certitude qu'ils étaient tous opposés à l'Union. Nous pouvons même affirmer qu'ils redoutaient la chose avant même que le projet ne prît corps. Dès 1837, Mgr Lartigue avart prévu, comme conséquence de nos troubles politiques, l'Union des Canadas; et il mit tout en œuvre pour empêcher ce malheur. De là la requête de fidélité à la Reine 
qu'il fait signer par le clergé de Montréal, en décembre, et où l'on peut lire ce qui suit:

Vos pétitionnaires osent espérer et en même temps supplier très humblement Votre Majesté, que les heure ıx habitants de cette colonie ne soient pas privés, pour le crime de quelques-uns, des avantages et privilèges, dont ils ont joui jusqu'à présent sous l'Empire Britannique ${ }^{1}$.

Et la preuve que le malheur redouté par Mgr Lartigue, c'était l'Union, se trouve dans la lettre qu'il adressait à Gosford, au début de février 1838 et dont voici le contenu:

Ne sachant pas le jour de son prochain départ pour la Grande-Bretagne, il lui envoie l'adresse que le clergé de Montréal présente en hommage à la Reine, en l'assurant que son attachement est fondé non sur la politique, mais sur la religion "qui nous fait un dogme immuable de la fidélité aux Puissances établies par l'ordre de Dieu sur la société". Il regrette la fin de son gouvernement paternel, juste et prudent, mais ses profondes connaissances du pays empêcheront, espèret-il, le gouvernement impérial de changer la constitution du Canada, et surtout l'Union des deux Provinces, "parce qu'elle amènerait en peu de temps pour l'Angleterre la séparation de ses colonies d'Amérique". Qu'il prévienne le Bureau Colonial de ne rien décider avant d'avoir reçu la pétition qui se signe dans le Bas-Canada pour s'opposer à cette Union des deux Provinces, que veut secrètement le même parti qu'en $1822^{2}$.

En octobre 1838, une requête portant la signature de Mgr Signay, de Mgr Turgeon et de Mgr Lartigue, où il est facile de reconnaître la pensée et l'influence de celui-ci, était présentée à Lord Durham. Son but essentiel est le maintien de l'allocation aux missions in-

1. Lettres, Mandements du diocèse de Montréal, 1: 23-24. Cf aussi Mandements des évêques de IQuébec, III: 377: Circulaire au sujet d'une adresse du clergé au parlement impérial, 7 février 1838; pp. 378-381, Adresse du clergé du diocèse de Québec au Parlement Impérial contre le projet d'unir le Bas et le Haut-Canada sous une même législature. - Le 20 septembre, Mgr Signay remercie lord Gosford d'avoir présenté cette pétition à la Reine et au Parlement. RAPQ (1938-1939), 274.

2. RAPQ, 1945-1946, 47. - La pétition contre l'Union dont parle ici Mgr Lartigue ne semble pas avoir eu de succès. La nomination et l'arrivée de Durham comme commissaire-enquêteur en auraient arrêté le cours. 
diennes, dont la suppression avait été décidée à Londres. On y lit pourtant le paragraphe suivant:

Vos pétitionnaires croiraient encore manquer à ce qu'ils doivent à Sa Majesté et à leur patrie, s'ils ne profitaient de l'occasion présente pour faire part à Votre Excellence des appréhensions qu'ont fait naître parmi les habitants catholiques du pays certains projets de lois qu'on prétend être actuellement médités et préparés par quelques agents du gouvernement, lesquels projets tendraient à affecter les lois, les usages, la langue et les autres droits religieux et civils, qui sont garantis aux Canadiens par les Traités et par le Statut du Parlement Britannique passé en la 14ème année du règne de Georges III, c. 83, particulièrement un plan d'éducation pour la jeunesse de la colonie.

Les évêques réclament des écoles catholiques distinctes, dans lesquelles le français sera la langue principale, l'anglais la langue secondés.

L'occasion de ce paragraphe, c'est le plan d'éducation rêvé pour le Bas-Canada par Arthur Buller. Mais comment ne pas reconnaitre que les évêques ont saisi du premier coup les conséquences du plan de Buller et protesté d'avance contre les injustices que le bill d'Union allait réserver au Bas-Canada?

Conséquence du fameux rapport Durham, le bill d'Union est introduit à la chambre des Communes au début de mai 1839. Le Canadien du 2 août en publie le texte intégral. Étienne Parent, par mesure de prudence, attend que les journaux de langue anglaise se soient prononcés avant de prendre position. Il écrit pourtant:

Pour nous mettre en garde contre une décision dont nous pourrions nous repentir, nous Canadiens Français, nous ne devons pas perdre de vue, dans la considération de cette grande question, que quoi qu'il arrive, la perte de notre nationalité est assurée; que nous soyons unis au Haut-Canada, ou que nous ne le soyons pas, il a été décidé en Angleterre que le BasCanada ne pouvait pas rester français.

3. RAPQ, 1938-1939, 282. 
La question nationale ainsi mise de côté, il reste à étudier les avantages et les désavantages de l'Union, du point de vue politique. Et Parent continue:

N'y aurait-il pas lieu d'espérer que les Canadas réunis cesseraient d'être le jouet des partis en Angleterre, et qu'ils obtiendraient plus facilement un Gouvernement satisfaisant, responsable, condition indispensable de bonheur et de prospérité pour eux? Cela vaudrait bien des pounds, des shillings et pence. Au reste, nous ne décidons rien, nous délibérons ${ }^{4}$.

Et il ouvre les colonnes de son journal à la discussion. On sait que la chambre des Communes reporta à la session de 1840 la discussion finale sur l'Union. Dans le Bas-Canada, personne, semble-t-il, ne songe à profiter de cette circonstance pour créer un mouvement populaire d'opposition. Quand Poulett Thomson, le nouveau gouverneur général arrive, mi-octobre 1839, Parent l'assure qu'il sera "à sa grande surprise peut-être" reçu avec sympathie et respect dans le Bas-Canada. Après une halte de quelques jours à Québec, Thomson se rend à Montréal pour y présider la réunion du Conseil Spécial, 11-14 novembre. La rumeur circule que le Conseil s'est déclaré favorable au principe de l'Union,mais aucune déclaration officielle ne vient appuyer ou démentir ce bruit ${ }^{5}$. Thomson chante son triomphe dans une dépêche à Russell ${ }^{6}$ et se rend à Toronto, où il a convoqué la réunion des Chambres pour le 3 décembre.

Il ne fallait rien moins que la discussion publique sur le sujet à la chambre d'Assemblée du Haut-Canada, les exigences, les explosions de fanatisme des extrémistes pour réveiller l'opinion populaire dans le Bas-Canada. Parent, qui commence à se ressaisir, écrit, le 23 décembre 1839, que non seulement les conditions de l'Union sont injustes, mais que l'Union elle-même est injuste. Elle l'est,

4. Le Canadien, 2 août 1839.

5. Au Canada, on ne connut qu'en février 1840 les résolutions votées par le Conseil Spécial et le nom des conseillers qui s'y opposèrent. Le Canadien du 17 février publie le texte de ces résolutions "lesquelles ont été publiées pour la première fois dans les journaux de Londres et qu'on n'a pas laissé transpirer" nous ne savons par quelles raisons", dit la Gazette de Montréal elle-même. Parent y va de sa réflexion et trouve le procédé "très gracieux".

6. Scrope, Memoir of the life of the Right Honourable Charles Lord Sydenham, Londres (1844), 135. 
ajoute-t-il, parce qu'elle dérange des "arrangements sociaux", que la Métropole nous a garantis, il y a cinquante ans et que, par la bouche de lord Gosford, elle s'était engagée solennellement à respecter, il y a quatre ans à peine. Si nous ne protestons pas hautement, c'est que comme Canadiens-Français, nous ne le pouvons pas. Mais si l'élément anglais de cette province qui croit l'Union injuste veut protester, nous nous unirons à lui. Son vœu est exaucé: le 17 janvier 1840 se tient à Québec, sous la présidence de John Neilson, la première assemblée antiunioniste du Bas-Canada. Et Parent est là. Ainsi, jusqu'à la mi-janvier 1840, il n'existe dans le BasCanada aucun mouvement organisé au projet d'Union.

\section{III}

Pendant ce temps que pensent et que font nos évêques? Le 13 avril 1839, après le rapport Durham, mais avant la présentation du projet d'Union à la chambre des Communes, Mgr Signay fait allusion au sujet dans une lettre à Mgr Provencher: doute sérieux sur l'efficacité de la mesure et résignation qui ressemble à celle d'Êtienne Parent, telle nous apparaît sa pensée?

Mgr Lartigue est plus ému et moins résigné. Il écrit:

Lord Durham a fait, en arrivant en Angleterre, un rapport à la Reine, très mauvais pour les Canadiens, qui demande l'Union des deux Canadas, le changement de nos lois civiles et même de notre langue pour les affaires politiques; il paraît même vouloir bouleverser notre éducation; ce qui influerait beaucoup sur la Religion ${ }^{8}$.

Le 27 avril, il s'adresse à Mgr Griffiths, Vicaire-Apostolique de Londres, et comme tel, défenseur des intérêts de l'Église auprès du gouvernement impérial. Il lui signale le rapport Durham qui a pour but de nous anglifier, i.e. de nous décatholiciser par une Union législative et par un système d'écoles neutres?

7. RAPQ, 1938-1939, 308.

8. A Mgr Provencher, 21 avril 1839. RAPQ, 1945-1946, 95.

9. RAPQ, 1945-1946, 97. 
Le 17 juin, après l'introduction du bill à la chambre des Communes, nouvelle lettre à Mgr Griffiths. Il parle des efforts que l'on fait pour priver les Canadiens de leurs lois, de leur langue, de leur religion.

Lord Durham depuis son retour en Angleterre a beaucoup aidé à cette disposition des esprits, en présentant à la Reine un rapport affreux contre les Canadiens, où les torts de notre peuple sont prodigieusement exagérés et dénaturés, et où il déploie tout son projet de nous anglifier, c'est-à-dire de nous décatholiciser, en procurant l'union législative du Haut et du Bas-Canada, pour nous dépouiller peu à peu de nos droits, surtout par son projet des écoles qui ôterait au clergé catholique de ce pays toute influence sur l'éducation de la jeunesse.

Il souhaite que tous les amis de la religion en Angleterre empêchent l'Union des Canadas et l'adoption d'une loi sur l'éducation des Canadiens, car "les bons règlements ainsi que les moyens d'éducation saine, libérale et religieuse ne manquent pas chez nous". Enfin, il demande si une requête du clergé à la chambre des Lords aurait quelque chance de succès ${ }^{10}$.

Le lendemain, c'est à lord Gosford qu'il écrit. Il lui demande de travailler à paralyser en Angleterre les préjugés de la presse anticanadienne, d'empêcher "l'union législative des deux Canadas qui, je le crains fort et j'en suis convaincu, amènerait avant dix ans la séparation de ces provinces d'avec Ja Mère-Patrie" et de faire en sorte que le parlemant impérial ne prive pas le Bas-Canada "de ses lois auxquelles il est très attaché, surtout à celles qu'il a adoptées pour l'éducation du pays, et qui conviennent mieux que toutes autres au génie et aux usages de notre peuple ${ }^{11 "}$.

Après ces démarches inspirées à Mgr Lartigue par ses devoirs d'évêque, il y a comme un temps mort; et il faut attendre jusqu'au 12 décembre pour retrouver dans sa correspondance l'angoissant problème de l'Union. Que s'est-il passé dans l'intervalle ? Deux faits, croyons-nous, ont pu atténuer les craintes de Mgr Lartigue et l'amener à suspendre son jugement. Le premier, c'est le billet que lui
10. RAPQ, 1945-1946, 103.
11. RAPQ, 1945-1946, 104. 
adressait de Londres, le 31 août 1839, Mgr McDonnell, évêque de Kingston:

Cette lettre sera remise à Votre Grandeur par l'honorable Poulett Thomson, le nouveau Gouverneu -Général du Canada. Il se rend dans le pays, j'ai tout lieu de le croire, favorablement disposé envers les catholiques. J'espère que Votre Grandeur aussi bien que votre clergé lui payeront toutes les attentions et lui rendront toutes les assistances dans leur pouvoir pour lui faciliter l'administration de son Gouvernement. En ce faisant, vous obligerez infiniment, de Votre Grandeur, le très humble et très obéissant serviteur ${ }^{12}$.

Mgr Lartigue pouvait-il regarder comme dénuée de tout fondement l'affirmation d'un ami, son confrère dans l'épiscopat, qui devait savoir, puisqu'il était à Londres même et en très haute réputation auprès du Ministre des colonies et qui, il va de soi, n'avait aucun intérêt à dénaturer les faits?

Le deuxième fait qui put atténuer les craintes de Mgr Lartigue, c'est l'élargissement d'Augustin-Norbert Morin. Sous le gouvernement de Colborne, Morin avait jugé prudent - et qui oserait l'en blâmer? - de rester caché. A peine le "vieux brûlot" est-il parti que Morin reparaît au grand jour et se met spontanément à la disposition de la justice. Incapable de comprendre ce qu'il y avait de loyauté et de grandeur dans ce geste, le procureur-général Ogden, une créature de Colborne, enjoint à Morin de choisir soit l'exil, soit la prison. Il opte pour celle-ci et il est écroué à Québec. Quelques jours plus tard, Thomson, après avoir étudié le dossier, ordonne l'élargissement de Morin sans conditions. Ainsi un cas que la justice Colbornienne juge digne d'exil n'offre même pas matière à procès pour la justice Thomsonnienne. L'écart est immense de l'une à l'autre et le bénéficiaire en est un Canadien Français et un catholique. Mgr Lartigue y vit-il une confirmation des bonnes dispositions annoncées par Mgr McDonnell? Nous ne le savons pas. Il eut du moins la prudence de ne pas se compromettre par des déclarations publiques et nous ne tarderons pas à le revoir au combat.

12. Archevêché de Montréal, cart. Toronto, 1838-1843. Cette lettre ne fut pas remise à Mgr Lartigue par Thomson lui-même comme l'avait pensé Mgr McDonnell. En effet, le gouverneur était encore à Québec quand Mgr Lartigue lui écrit dans le sens suggéré par Mgr McDonnell. RAPQ, 1945-1946, 117. 
Il faut rendre ce témoignage aux journalistes canadiens-français de l'époque, à Étienne Parent le premier, que tout en appréciant le geste de Thomson à l'endroit de Morin, ils l'ont regardé comme un acte de justice, non comme une grâce. Ils se réservent de juger le gouverneur à ses œuvres; en quoi ils faisaient preuve de sagesse. Car c'était en de longues conversations avec Durham que Thomson avait appris à connaître les Canadiens Français. Deux jours après son arrivée à Québec, il écrit que le Bas-Canada est, pour le moment, tranquille, parce qu'il est faible, qu'il ne peut plus compter sur le secours des États-Unis; mais il lui prête encore de noirs desseins d'insubordination et de révolte. Cet homme, qu'on nous dit envoyé au Canada pour se renseigner et renseigner la Métropole relativement à l'Union, n'a pas besoin de renseignements. Cette dépêche de Thomson à Russell n'est rien autre chose qu'un écho de ses conversations avec Durham. Car en deux jours, il n'a guère eu le temps de consulter, d'observer, il n'est guère sorti de son palais; et il est a Québec, resté indéfectiblement loyal à l'Angleterre avant, pendant et après les Troubles de 1837.

On avait pu entourer d'un certain secret les délibérations du Conseil Spécial de Montréal. Mais les discussions de la Chambre du Haut-Canada étaient publiques; l'on ne tarda pas à voir où Thomson voulait en venir. Et l'angoisse étreignit de nouveau l'âme de $\mathrm{Mgr}$ Lartigue.

Le 12 décembre, il écrit à Gosford. Après l'avoir remercié de la part qui lui revient dans l'obtention des lettres patentes de l'évêrhé de Montréal, il s'élève de nouveau contre l'Union des Canadas, parce qu'elle est contraire aux privilèges déjà accordés aux colonies par la Grande-Bretagne, parce qu'elle sera accompagnée d'injustices criantes en faisant, payer les dettes du Haut-Canada par le Bas-Canada, en transportant le siège du gouvernement dans la province supérieure. "Un peuple a mille lieues de chez vous ne tiendrait à de pareils outrages; et vous ne pourriez guère compter sur sa fidélitét $e^{13}$."

Nous avons vu que le ton passionné de la chambre d'Assemblée du Haut-Canada avait été pour Étienne Parent comme une première occasion de se ressaisir. Nous connaissons également la réac-

13. RAPQ, 1945-1946, 121. 
tion de Mgr Turgeon. Le 28 décembre, il écrit à l'honorable Dominique Daly, secrétaire provincial, que le clergé de Québec est grandement allarmé de ce qui se passe actuellement dans le Haut-Canada. Nous avons de très grandes raisons de croire que la mesure favorite de l'administration actuelle, si elle est effective, nous conduira à de nouveaux malheurs et à des malheurs irréparables. Vous savez que les patriotes l'acceptent, tout en la regardant comme injuste, et les motifs de cette acceptation vous sont connus, je n'en doute; mais j'appréhende très fort que ceux qui ont aujourd'hui en leurs mains le sort des Canadas ne se fassent illusion sur ces motifs et ne veuillent apprécier à sa juste valeur l'apathie actuelle et seulement apparente de nos agitateurs. Je prie Dieu bien sincèrement qu'il nous préserve d'une Union qui, selon mon avis et celui de bien d'autres, nous conduirait à l'Union républicaine, notre voisine ${ }^{14}$.

La preuve est faite, croyons-nous, Mgr Signay, Mgr Turgeon et Mgr Lartigue redoutent l'Union; ils lui sont fortement opposés. Mgr Bourget n'est pas d'un autre sentiment, comme le prouvent les entretiens qu'il eut avec Thomson sur le sujet ${ }^{15}$. Et nous sommes ainsi arrivés au début de 1840, époque où l'opposition à l'Union prend corps dans le Bas-Canada. Quelle sera l'attitude de nos chefs ecclésiastiques ? Que feront-ils? Si les évêques de Québec et de Montréal s'entendent sur l'opportunité, voire la nécessité de favoriser ce mouvement, de faire des représentations au parlement impérial, des circonstances indépendantes de leur volonté ne leur permettent pas de s'unir dans une même protestation. Force nous est donc de raconter en des paragraphes distincts ce qui se fit à Québec et ce qui se fit à Montréal.

\section{IV}

Des lettres de Mgr Turgeon à Mgr Bourget, conservées à l'archevêché de Montréal, il ressort qu'il y eut dès le début à Québec, des relations très étroites entre l'évêché et le comité présidé par John Neilson. C'est le 17 janvier 1840 qu'a lieu la réunion préliminaire où sont arrêtées les résolutions qui entreront dans l'adresse ou pétition au parlement impérial contre l'Union. Le 24 janvier, elles sont communiquées à une assemblée publique qui les accueille avec

14. RAPQ, 1938-1939, 356.

15. Mgr Bourget à Mgr Signay, 21 février 1840. RAPQ, 1945-1946, 216. Même au même, ibid, 218. 
enthousiasme. Et le lendemain, 25 janvier, Mgr Signay adresse une circulaire à son clergé:

Je n'hésite pas à vous déclarer que l'on vous verra avec plaisir user prudemment de votre influence auprès de vos paroissiens pour les engager à signer l'adresse qui va leur être présentée, et dont le succès, comme il est à prêsumer, dépendra du nombre de signatures dont elle sera revêtue ${ }^{16}$.

Le clergé était par là-même autorisé à signer la requête et il le fit. Que penser de ce geste de Mgr Signay? Il s'adresse, non au peuple, mais à son clergé; ce n'est pas un ordre qu'il donne, mais une invitation qu'il fait; encore y faut-il de la prudence dans l'exécution. Le mouvement d'opposition a pris naissance chez les laĩques; il exprime les sentiments de l'immense majorité des habitants tant de langue française que de langue anglaise et du district de Québec et du district des Trois-Rivières, qui dépend de l'évêché de Québec. Dans ces circonstances, Mgr Signay et son clergé pouvaient difficilement rester à l'écart; en effet, leur abstention aurait été interprétée comme une approbation de l'Union. Pouvaient-ils davantage?

Présenter une requête comme corps distinct? Nous affirmons, sans crainte de nous tromper, qu'une telle requête n'aurait pas pesé plus lourd que les autres. Après le 25 janvier, date de la circulaire de Mgr Signay à ses prêtres, il aurait été nuisible au bien de la cause et même à la bonne réputation du clergé de faire machine arrière ou d'adopter un autre mode de protestation. Et avant cette date, les laĩques auraient-ils lancé leur requête s'ils n'avaient pu compter sur l'appui total du clergé?

Et donc dans les requêtes de Québec et des Trois-Rivières que l'envoyé du comité Neilson porte à Londres et qui sont revêtues de 39,028 noms, les signatures du clergé voisinent avec celles des laïques $^{17}$.

\section{$\mathrm{V}$}

L'atmosphère de Montréal, en ce début de 1840, était loin d'être celle de Québec. Québec n'a pas ou presque pas souffert des Troubles de 1837 et de 1838; Montréal en a terriblement souffert, il en est

16. Mandements... des évêques de Québec, III: 400.

17. Le Canadien, ler mai 1840, reproduit de la Gazette de Québec le tableau par comtés des signatures contre l'Union, dans les districts de Québec et des TroisRivières. - Pour être complet, il faudrait parler de la part des évêques dans la sous_ 
encore ému, abattu, aigri. Québec n'a pas ou a été peu éprouvé dans la personne de ses chefs politiques. Un homme comme John Neilson n'a rien perdu de son prestige et sa vaste expérience des hommes et des choses inspire confiance. Qu'il se lève et on marchera à sa suite; c'est ce qu'a fait Étienne Parent et on sait ce qui en est résulté. Montréal n'a plus de chefs politiques: les uns sont morts sur l'échafaud, les autres sont en exil ou en prison. C'est en 1841 que LaFontaine prendra vraiment figure de chef; en 1840, il n'est rien autre chose qu'un lieutenant sans général; son mérite et sa valeur ont été éclipsés par la puissante personnalité de Papineau.

Et du reste, que pensent de l'Union ces vaincus de 1837 ? Nous avons entendu Mgr Turgeon déclarer qu'ils la croyaient injuste, mais qu'ils n'osaient pas manifester leur sentiment pour des raisons à eux. Nous retrouvons la même pensée sous la plume de Mgr Bourget écrivant à Mgr Signay:

Il est à croire que nos Laïcs ne voudront point se remuer pour éviter le malheur de l'Union. Plusieurs de nos Patriotes sont sous cette impression que l'Union des deux Provinces opérera plus vite leur désunion de la Mère-Patrie ${ }^{18}$.

Atmosphère de Montréal si peu claire et si peu encourageante que le comité de Québec, très actif et très ardent chez Jui, ne cherche pas à pénétrer dans la métropole. Celui qui souffre le plus de cette inertie, c'est Mgr Lartigue. Dès la réception de la circulaire de Mgr Signay, il avait persé soit la faire sienne, soit en publier une semblable. Il attend que l'opinion publique s'émeuve et l'attente lui paraît terriblement longue. Le 10 février, soit trois semaines après la première réunion de Québec, il écrit à $M$. Mignault, curé de Chambly, qu'il ne voit jour à publier sa circulaire au clergé; l'apathie est générale, tant à la ville qu'à la campagne. "Quant au clergé,

cription organisée pour défrayer les dépenses de l'envoyé à Londres, M. V. Testu. Mgr Turgeon se fait le solliciteur du comité de Québec auprès du clergé et des communautés religieuses. Il écrit à Mgr Bourget, le ler mars qu'il faut recueillir la somme de L750. Il a souscrit L10, Mgr Signay, L25, les trois communautés de Québec, L10 chacune. La souscription des curés à revenus modiques, tels les curés de l'AngeGardien et des deux Lorette est de L10. Mgr Bourget lui permet de faire appel à la charité du clergé de Montréal. RAPQ, 1945-1946, 219. Mgr Lartigue et Mgr Bourget qui avaient pourtant leurs propres soucis, voulurent contribuer personnellement à la souscription de Québec.

18. RAPQ, 1945-1946, 216. 
ajoute-t-il, il est difficile qu'il se présente seul à la brèche dans cet assault19."

Enfin, le 21 février, un mois après Québec, Montréal a sa première assemblée anti-unioniste, présidée par F.-X. Mailhot. Après lecture du texte de la requête au parlement impérial, faite par La Fontaine, Charles Mondelet propose, appuyé par L.-C. Drummond, un texte plus conciliant et suivant lequel le Bas-Canada accepterait une Union qui lui rendrait pleine et entière justice. Côme-Séraphin Cherrier et Olivier Berthelet proposent l'adoption du texte de La Fontaine et l'amendement de Mondelet est rejeté. Il marque, du moins, que l'union des esprits n'est pas parfaite ${ }^{20}$.

De plus, le comité de Montréal ne marche pas la main dans la main avec l'évêque. Si l'on en croit l'Ami du Peuple du 28 mars, avant de se manifester au grand jour, le comité de Montréal aurait envoyé des éclaireurs auprès de Mgr Lartigue afin de solliciter son appui. Celui-ci aurait voulu connaître le texte de la requête avant de promettre sa collaboration. Ce qui nous parait sagesse parut exagération et l'on décida de se passer de l'évêque.

En lisant l'adresse du comité de Montréal au parlement impérial, Mgr Lartigue pensa qu'il avait eu raison de ne pas se compromettre avant l'heure. Il repoussait l'Union de toutes les forces de son âme. Mais, intransigeant comme toujours devant ce qui lui paraît erreur, il n'admet pas qu'on ait recours à des arguments repréhensibles. Il avait vécu intensément la période de 1837; il en avait souffert dans son âme autant sinon plus que tout autre. Et il n'arrivait pas à se convaincre qu'il fallait faire peser uniquement sur les mauvaises administrations coloniales la responsabilité de tous nos malheurs. Ce paragraphe de la pétition de Montréal lui paraissait non seulement malhabile, mais historiquement inexact. Il ne pouvait, en conscience, y apposer sa signature et il n'entendait pas que son clergé le fit $^{21}$. Ainsi il ne peut signer la requête du comité de Montréal; d'autre part, il ne peut garder le silence. Dès lors, il décrète que le clergé du diocèse aura, comme corps distinct, sa propre pétition contre l'Union; et Thomson lui-même, qui affecte d'être scandalisé

19. RAPQ, 1945-1946, 128.

20. Le Canadien du 28 février reproduit le compte rendu de la réunion de Montréal, d'après l'Aurore des Canadas.

21. Circulaire au clergé, 27 février 1840. RAPQ, 1945-1946, 128=12:4 
en voyant le clergé de Québec s'unir aux laĩques, admet la légitimité de ce procédé.

Mgr Lartigue rédige lui-même le document, dont voici l'essentiel:

La séparation gouvernementale des deux provinces est devenue bien plus nécessaire depuis que, par l'union des deux provinces canadiennes, projetée contre les traités et les actes solennels de la Grande-Bretagne, nous sommes menacés de perdre les droit: acquis et garantis en faveur de notre religion, de nos lois et même de notre langue: que cette union n'aurait lieu qu'à des conditions avilissantes et onéreuses pour ce pays, telles que d'aider à payer les dettes du Haut-Canada que nous n'avons jamais contractées, d'être défranchisés de nos droits d'électeurs dans la proportion qui nous est due comme sujets Britanniques, et même de voir transporter le siège du Gouvernement, qui est la résidence naturelle d'un Gouverneur-Général du Bas au Haut-Canada.

Une pétition semblable à celle-ci, signée en 1822 par 87,000 sujets fidèles de Sa Majesté, préserva alors cette province d'un malheur semblable, parce que les illustres rois, vos ancêtres, se rappelèrent alors des exploits des Canadiens pour la défense constante du pays et pour la gloire des armées Britanniques, au lieu de punir une Province entière pour la faute d'un petit nombre proportionnel de coupables.

C'est pourquoi vos pétitionnaires supplient très humblement que le Gouvernement leur soit rendu, tel qu'il existait dans cette Province, en vertu de l'acte de la 31ème année de Geo. III, c. 31 , et ferez justice ${ }^{22}$.

Cette requête, signée par tous les prêtres du diocèse de Montréal, s'adressait aux trois branches du parlement impérial, i.e. à la Reine et à son conseil, à la chambre des Lords et la chambre des Communes. Il était dans l'ordre que la copie destinée à la Reine lui fût envoyée par le gouverneur-général et. Thomson voulut bien s'en charger ${ }^{23}$. Les copies destinées à la chambre des Lords et à la cham-

22. RAPQ, 1945-1946, 129.

23. Mgr Lartigue à Thomson, 31 mars 1840, RAPQ, 1945-1946, 133. Mgr Bourget eut la naìveté de croire que Thomson appuierait la requête du clergé. L'illusion était de taille! Car c'est exactement le contraire qui arriva. Cf Rapport sur les Archives Publiques pour l'année 1942, p. 71; Thomson à Russell (No 80), 4 avril 1840: "Il lui transmet une pétition du clergé catholique romain du Bas-Canada qui désapprouve l'union et demande qu'on rétablisse la constitution. Il ne croit pas qu'on doive porter beaucoup d'attention à cette adresse. On devra en accuser réception; les adresses laissées sans réponse dans le passé ont causé du dépit." 
bre des Communes étaient adressées à Mgr Griffiths pour être transmises, l'une à Gosford, l'autre à O'Concell'24. En même temps, Mgr Lartigue écrit à l'un et à l'autre et il leur demande de vouloir bien s'opposer érergiquemert au bill de l'Union. Il insiste auprès de Gosford sur le danger auquel s'expose l'Angleterre de perdre le Carada, auprès d'O'Concell sur les dangers que présente l'Union au point de vue religieux ${ }^{25}$.

Ces beaux efforts étaient voués à l'insuccès. L'Union avait été décidée; elle fut adoptée, sanctionnée, imposée. Mgr Lartigue ne devait pas connaitre ici-bas l'issue de cette lutte, dans laquelle il avait, costre vents et marées, si vaillamment combattu. Il mourait à l'Hôtel-Dieu de Montréal, le 19 avril 1840 .

Nos évêques, et c'est la leçon qui se dégage de cette histoire, avaient plus de sagesse et de clairvoyance que les Durham, les Russell et les Thomson. Si l'Union des Canadas a produit quelque bien, c'est précisément dars la mesure où elle n'a pas été l'Union, i.e. où elle s'est libérée de l'esprit qui l'avait inspirée et contre lequel ont lutté Jes évêques. Et quand, après un quart de siècle, elle disparaît pour faire place à un autre mode de gouvernement, la preuve est faite depuis longtemps qu'elle n'avait pas toutes les vertus que lui avajent si généreusement reconnues ses pères. En effet, le bill d'Union n'était pas encore sanctionné que LaFontaire, Baldwin et Hincks se rencontraient à Toronto. Puisqu'ils re pouvaient pas empêcher l'Union, ils avisaient aux moyens pratiques d'en corriger les erreurs et les injustices ${ }^{26}$.

Léon Pouliot, s.j.

24. RAPQ, 1945-1946, 12 mars 1840, 131-132.

25. RAPQ, 1945-1946, 132. - Sur l'intervention de Gosford et d'O'Connell contre l'Union, ef Chapais, Cou's d'histoire d: C'anada, IV: 301 et ss.

26. Le bill d'Union fut sanctionné le 23 juillet 1840 Or, Le Canadien du 3 juillet publiait la note suivante: "M. L.-H. La Fontaine est parti ces jours derniers pour aller à Toronto. Le Herald prête un but politique à ce voyage. Selon les informations desce journal, M. La Fontaine "doit prendre des arrangements avec M. Baldwin, Solliciteur Général de Sa Majesté, et d'autres rebelles suspectés ou connus, relativement à la tactique à suivre dans l'assemblée des provinces unies." Les informations du Herald étaient exactes quant au but du voyage de La Fontaine. 